\title{
Obtención de materiales híbridos homogéneos de tipo Orgánico/Inorgánico
}

\section{Obtaining homogenous Organic / Inorganic hybrid materials}

\author{
RAMÍREZ-SALAS, Virginia ${ }^{1} \uparrow$, MORENO-MARTINEZ, Beatriz Eugenia ${ }^{2 *}$, ALARCÓN-RUIZ, Erika $^{1}$ \\ y ORDOÑEZ-PACHECO, Luis Daniel ${ }^{1}$
}

${ }^{I}$ Tecnológico Nacional de México. Instituto Tecnológico de Ciudad Madero

${ }^{2}$ Tecnológico Nacional de México. Instituto Tecnológico de Nuevo León

ID 1 ${ }^{\text {er }}$ Autor: Virginia, Ramírez-Salas / CVU CONACYT ID: 173197

ID $1^{\text {er }}$ Coautor: Beatriz Eugenia, Moreno-Martínez / CVU CONACYT ID: 162379

ID $2^{\text {do }}$ Coautor: Erika, Alarcón-Ruiz / CVU CONACYT ID: 163514

ID $3^{\text {er }}$ Coautor: Luis Daniel, Ordoñez-Pacheco / CVU CONACYT ID: 844011

DOI: 10.35429/JCPE.2019.20.6.14.19

\begin{abstract}
Resumen
Los polímeros tridimensionales son mutuamente incompatibles y una solución es la formación de una red polimerica interpentrada. Para obtener placas ópticamente transparentes, se sintetizaron las redes individuales de poliuretano (PU), polimetilmetacrilato (PMMA) y sílica $\left(\mathrm{SiO}_{2}\right)$ y se obtuvieron mejores condiciones de reacción para cada material. Se obtuvieron redes binarias y ternarias con diferentes porcentajes de sílica, incorporando al sistema el $10 \%$ en peso de sílica, llevándose a cabo una polimerización in situ en masa. El interés de este trabajo radica en la asociación de una red inorgánica $\left(\mathrm{SiO}_{2}\right)$ a dos redes orgánicas PU y PMMA. Para reducir la separación de fases que ocurre en el sistema PU, PMMA y $\mathrm{SiO}_{2}$ se introdujeron agentes acoplantes de naturaleza organico/inorgánico. Se obtuvieron placas ópticamente transparentes utilizando el trimetoxisilil propil metacrilato y el isocianotopropil trietoxi silano. Los materiales se caracterizaron por Espectroscopia Infrarroja por transformada de Fourier verificando la presencia de los grupos funcionales representativos en cada una de las muestras. El propósito de incorporar estos materiales a una sola red es mejorar las propiedades mecánicas, ópticas y térmicas de los polímeros.
\end{abstract}

IPN’s, Redes poliméricas interpenetradas
Recibido 09 Junio, 2019; Aceptado 28 Septiembre, 2019

\begin{abstract}
Tridimensional polymers are mutually incompatible and a solution is the formation of interpenetrating polymer networks (IPN's). In order to obtain optically transparent plaques, the polyurethane (PU), polymethylmetacrylate (PMMA) and sílica $\left(\mathrm{SiO}_{2}\right)$ were syntetized and we obtained the best conditions of reaction for each material. We sintetized the binary networks and the ternary ones with different percentage from sílica were obtained, incorporating to the system $10 \%$ in weight of sílica, carrying out a curing in situ in mass. The interest of this work lies in the association of an inorganic network $\left(\mathrm{SiO}_{2}\right)$ to two organic networks PU and PMMA. In order to reduce the phase separation that occurs in the PU, PMMA and $\mathrm{SiO}_{2}$ system, coupling agents of organic/inorganic nature were introduced. Optically transparent plaques were obtained using trimethoxysilyl propyl methacrylate and isocyanatopropyl triethoxy silane. The materials were characterized by Fourier Transformed Infrared Spectroscopy, verifying the presence of representative functional groups in each of the samples. The purpose of incorporating these materials to a single network is the improvement of the mechanical, optical and thermal properties of the organic polymers.
\end{abstract}

IPN's, Interpenetrating polymer networks

Citación: RAMÍREZ-SALAS, Virginia, MORENO-MARTINEZ, Beatriz Eugenia, ALARCÓN-RUIZ, Erika y ORDOÑEZPACHECO, Luis Daniel. Obtención de materiales híbridos homogéneos de tipo Orgánico/Inorgánico. Revista de Energía Química y Física. 2019. 6-20: 14-19

\footnotetext{
* Correspondencia al Autor (Correo electrónico: virginia.itcm.tecnm@gmail.com)

$\dagger$ Investigador contribuyendo como primer Autor
} 


\section{Introducción}

Los polímeros son, en su mayoría, termodinámicamente incompatibles entre ellos a causa de la desfavorable entropía de la mezcla. Se ha buscado la manera de asociar una red inorgánica con un polímero orgánico para la formación de materiales híbridos orgánicosinorgánicos homogéneos. La síntesis de la red de polímeros interpenetradas (IPN) es una valiosa estrategia para reforzar y mejorar las propiedades de un polímero red combinando una segunda red en ella (Liang y Deng, 2017). La primera síntesis de materiales minerales a partir de reacciones de polimerización mediante el uso de precursores moleculares de tipo alcóxido metálico fue realizada por Ebelmen en 1845, obteniendo con algunas gemas como el zafiro, rubí y esmeralda (Sanchez y col, 1996).

Este método de elaboración de materiales, se conoce como el "procedimiento sol-gel", suscitándose un fuerte interés de renovación tanto en la comunidad universitaria como en la industria. La presencia de compositos orgánicos permiten modificar las propiedades mecánicas, elaborar películas y fibras, obtener por simple moldeado diversas estructuras geométricas para óptica integrada, controlar la porosidad y ajustar el balance hidrófilo/hidrófobo de la red.

Por su parte los compositos inorgánicos pueden jugar varios papeles, tales como: contribuir a aumentar la tensión mecánica y térmica del material, permitir modular el índice de refracción, además de añadirle propiedades electroquímicas, magnéticas y eléctricas (Babonneau y col., 1996). Es por eso que a las IPN's que se forman de compositos orgánicos / inorgánicos logran tener excelentes propiedades ópticas y térmicas, recibiendo el nombre de materiales híbridos.

Ghosh and Karak, 2018 sintetiaron redes de polímeros interpenetradas resistentes (IPN) biodegradables e inteligentes de poliuretano, silicona y poliestireno, los cuales exhibieron buenas propiedades mecánicas, biodegradabilidad, resistencia al impacto, resistencia al rayado y buena estailidad térmica ademas de resitencia química.
Para la formación de la primera red se llevó a cabo una reacción de policondensación, haciendo reaccionar un poliol del tipo poliéster (aceite de ricino) (Dave, 2013) y un poliisocianato alifático basado en hexametilen diisocianato (HDI) del cual se obtiene un triisocianato (Desmodur N3600) y usando como catalizador de esta reacción del dibutil dilaureato de estaño (DBTL). En la formación de la segunda red, se utilizó el polimetilmetacrilato, que es un polímero amorfo, puesto que presenta una alta transparencia, además de una gran resistencia al impacto. Para la formación de la tercera red, se utilizó el tetraetilortosilicato (TEOS) iniciando la reacción con una solución de ácido clorhídrico.

Existen pocos antecedentes de materiales híbridos ternarios que hayan sido sintetizados del modo en el que se ha propuesto en esta metodología, en donde la sílica no se uso como carga sino que, el tetraetil ortosilicato fue el monómero de la tercera red de $\mathrm{SiO}_{2}$, formando un sistema híbrido PU/PMMA/ $\mathrm{SiO}_{2}$ sin y con agentes acoplantes que funcionalizarán las IPN's ternarias. La finalidad de este trabajo fue obtener materiales híbridos inorgánicos/orgánicos, realizando una polimerización en masa para el sistema ternario. Estos materiales juegan un rol importante en diversos sectores industriaeles como lo son en la automotriz, calzado, textil, aplicaciones biomédicas, optoelectrónica, etc.

En las siguientes secciones, se presentará la metodología para la preparación de cada uno de los materiales. La técnica de caracterización mediante FTIR, permitió verificar la presencia de los grupos funcionales caracterisicos de las IPN's, en donde se observaron los movimienetos de tensión, elongasión, vibrasión simétricas/asimétricas y deformaciones de las estructuras hidrocarbonadas. Los resultados fueron obenidos de un equipo Spectrum One marca Perkin Elmer e interpretados mediante tablas de elucidación de compuestos orgánicos/inorgánicos.

\section{Metodología a desarrollar}

\section{Síntesis de IPN's individuales y binarias}

Se sintetizaron las redes individuales de PU, PMMA y $\mathrm{SiO}_{2}$, asi como las redes binarias PU/PMMA, PU/ $\mathrm{SiO}_{2}$ y PMAA/SiO 2 . Una vez que se determinó la mejor relación en masa de los componentes para cada red binaria 
Se sitnetizaron las redes ternarias variando la composición en masa $(95 / 5,90 / 10$, $85 / 15$ y $80 / 20)$ asi como las ternarias (45/45/10 y 41/41/10 con $4 \%$ de IPTS y TSMA.

\section{IPN in situ SEC (secuencial)}

Se puede fijar una determinada separación de fase entre los dos procedimientos de cada una de las polimerizaciones. En este caso, los IPN sintetizados se calificarán como IPN in situ SEC (proceso secuencial) y este se describe en la figura 1 .

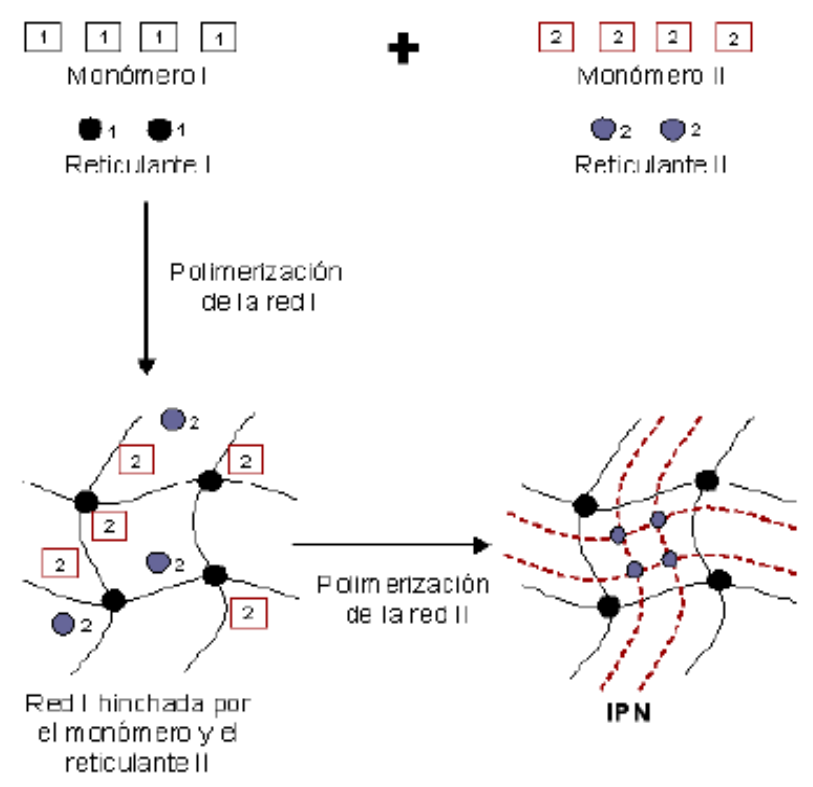

Figura 1 Esquematización del método in situ secuencial de una IPN

Fuente: Sperling (2002)

\section{IPN in situ SIM (simultánea)}

Se puede decir, que a partir de una mezcla inicial homogénea, se produce simultáneamente la síntesis de las dos redes. En estas condiciones las IPN sintetizadas se calificarán como IPN in situ simultánea (proceso simultáneo) figura 2.

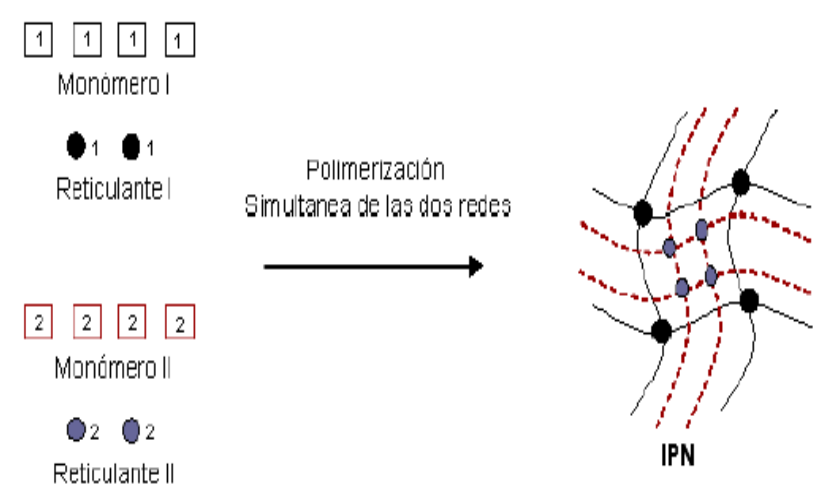

Figura 2 Esquematización del método in situ simultánea de una IPN

Fuente: Sperling (2002)

\section{Síntesis del sistema PU/PMMA/SiO 2 sin agentes acoplantes}

Con base en la mayor cantidad de TEOS incorporada en los sistemas binarios, se determinó la cantidad de sílica con la que se formó el sistema de tres redes, manteniendo una relación 45/45/10 en peso. Se mezclaron los monómeros (NCO, OH, MMA, TRIM y el TEOS) en un recipiente. Se agregó el iniciador de la red de PMMA y se homogenizó. Posteriormente, fue incorporado y mezclado el catalizador de la red de PU hasta homogenizar. Finalmente, se adicionó la solución acuosa de TEOS y $\mathrm{HCl}$, para la red de $\mathrm{SiO}_{2}$ y se homogenizó por última vez, colocando la muestra en un molde de vidrio y dejando reposar en un baño ultrasónico; se eliminaron las burbujas generadas por el $\mathrm{CO}_{2}$ liberado durante el proceso de reacción. La reacción se realizó en un baño a $60^{\circ} \mathrm{C}$ por 24 horas. La película obtenida, se llevó a estufa de vacío durante 24 horas a $80^{\circ} \mathrm{C}$ para eliminación de monómeros residuales.

\section{Síntesis del sistema PU/PMMA/SiO 2 con agentes acoplantes}

Los agentes acoplantes utilizados IPTS para el PU y el MSMA del PMMA, se agregaron en un $4 \%$ en peso máximo, calculado para la red ternaria; el proceso de síntesis para esta IPN, se realizó con los mismos pasos de la red sin acoplantes. Las mejores IPN's fueron seleccionadas para realizar las caracterizaciones mediante FTIR.

\section{Caracterización por Espectroscopía Infrarroja de Transformada de Fourier (FTIR)}

Las técnicas de caracterización como el FTIR, permiten la localización e identificación, virtualmente, de todos los grupos funcionales, pues muestran absorciones específicas y características en la región del infrarrojo. La espectroscopía en el infrarrojo mide los cambios en las vibraciones de estiramiento y flexión que ocurren cuando una molécula absorbe energía electromagnética. Los movimientos vibratorios posibles de una molécula dependen de dos cosas: 1) la masa de los átomos que forman un enlace dado y 2) la naturaleza del enlace en sí, esto es su energía de enlace. 
La frecuencia del movimiento depende no solo de la fuerza de los átomos que interviene, sino también del ambiente y de la molécula completa. En general, la absorción del IR se da entre 5000 y $1250 \mathrm{~cm}^{-1}$ se atribuyen a los modos de estiramiento vibratorio de diversos grupos funcionales. Los picos de absorción ente 1250 y $500 \mathrm{~cm}^{-1}$ del espectro IR generalmente son el resultado de una compleja combinación de excitación de vibración y de rotación de la molécula completa.

Gran parte de esta absorción se debe a la flexión que requiere menos energía que el estiramiento, hay numerosas direcciones en las que puede ocurrir una flexión leve, lo que da lugar a numerosas absorciones en el IR, (Wingroove, 2004). Los espectros se obtuvieron de un espectrómetro Spectrum One marca Perkin Elmer con una resolución de $2 \mathrm{~cm}^{-1}$ y 32 scan. Las muestras obtenidas se pulverizaron manualmente y fueron incorporadas en una pastilla de $\mathrm{KBr}$.

\section{Resultados}

Resultados de la sección 2

La concentración de la red híbrida resultante PU/PMMA/SiO ${ }_{2}$ es de 45/45/10, sin agente acoplante; la concentración máxima permitida de dichos agentes (IPTS y TSMA) para ser considerados como materiales híbridos es hasta de un $4 \%$ en peso total de la red ternaria, resultando una IPN ópticamente transparente en comparación con la red sin agentes. Para la preparación de los materiales se usó una relación molar constante de $\mathrm{NCO} / \mathrm{OH}=2.5$ y los resutlados de las IPN's sintetizadas se resumen en la tabla 1 .

\begin{tabular}{|c|c|c|c|c|c|}
\hline \multirow[b]{2}{*}{ PU } & \multicolumn{4}{|c|}{$\%$ en peso } & \multirow{2}{*}{ Aspecto } \\
\hline & PMMA & TEOS & $\begin{array}{c}\text { IPTS }_{(\mathrm{Ag}} \\
\mathrm{Ac} \text { PU) }\end{array}$ & $\begin{array}{c}\text { TSMA } \\
(\mathrm{Ag}, . \mathrm{Ac}, \mathrm{MMA})\end{array}$ & \\
\hline 100 & 0 & 0 & 0 & 0 & ts \\
\hline 0 & 100 & 0 & 0 & 0 & $\operatorname{tr}$ \\
\hline 0 & 0 & 100 & 0 & 0 & op \\
\hline 90 & - & 10 & - & - & $\operatorname{tr} / \mathrm{fx}$ \\
\hline 90 & - & 10 & - & - & ts/rg \\
\hline 45 & 45 & 10 & - & - & $\mathrm{op} / \mathrm{fx}$ \\
\hline 41 & 41 & 10 & 4 & 4 & $\mathrm{tr} / \mathrm{fx}$ \\
\hline
\end{tabular}

Tabla 1 IPN's sintetizadas a diferentes concentraciones de TEOS: op=opaca, $\mathrm{fx}=$ flexible, $\mathrm{tr}=$ transparente, $\mathrm{ts}=$ traslúcido, $r g=$ rígido

\section{Resultados de la sección 3}

En la figura 3, se observa muestra el espectro infrarrojo de la red ternaria incorporando al sistema orgánico una cantidad de TEOS de $10 \%$ del peso total calculado para la red y un $4 \%$ en peso de los agentes acoplantes. Los espectros mostrados representan los grupos funcionales característicos de la red ternaria sin agentes y con agentes acoplantes. En la región de los 3200$3670 \mathrm{~cm}^{-1}$ y $940-1002 \mathrm{~cm}^{-1}$, de ambos espectros, se encuentra las bandas atribuidas a los grupos $\mathrm{OH}$, éstas se originan por los $\mathrm{OH}$ 's del poliol y al silanol residual.
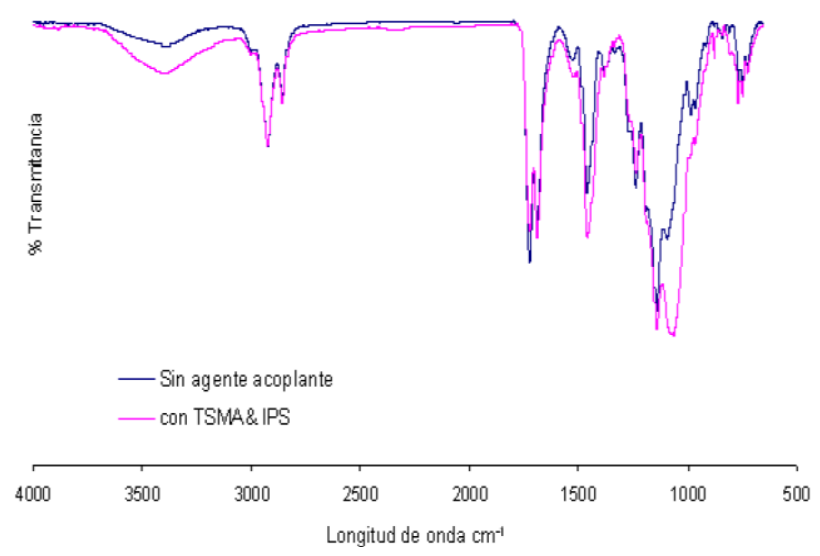

Figura 3 Espectro FTIR para el sistema PU/PMMA/SiO en un intervalo de $4000-500 \mathrm{~cm}^{-1}$

Fuente: Elaboración propia

A $1360 \mathrm{~cm}^{-1}$ de los dos espectros se localiza un pico que se atribuye a la posible formación de halogenuro de carbamilo generado por la reacción del ácido clorhídrico combinado con el isocianato usado en la formulación del $\mathrm{PU}$, además se encuentra una banda intensa que se atribuye al grupo uretano $\mathrm{N}-\mathrm{C}$ a $1460 \mathrm{~cm}^{-1}$. Además a 2854 y $2922 \mathrm{~cm}^{-1}$, se localizan los picos de los grupos metilos $\left(-\mathrm{CH}_{2}\right)$ y metilenos $\left(-\mathrm{CH}_{3}\right)$.

Los picos característicos del PMMA (C=O y C-O) a 1724 y $1140 \mathrm{~cm}^{-1}$, sílica, silanol residual, haluro de carbamilo y el enlace del grupo uretano N-H se esquematizan en la figura 4 con una escala de $1830-630 \mathrm{~cm}^{-1}$, observando una gran intensidad en las bandas del espectro sintetizado con agentes acoplantes. 
A $1685 \mathrm{~cm}^{-1}$ se presenta un pico que puede ser generado por la presencia de posibles reacciones secundarias tales como la poliurea dentro de la IPN en donde los grupos N-H son altamente reactivos frente a los isocianatos (Desmodur N3600), generando enlaces del tipo urea (amida $\mathrm{I}^{\mathrm{a}}$-HNCO-) y esta intensidad se ve en mayor proporción en el espectro que contiene IPTS, debido a que se funcionaliza la red de PU y PMMA con el TSMA; a $1083 \mathrm{~cm}^{-1}$ se presenta una banda fuerte, que se atribuye a la flexión del enlace C-O originada por la cadena del poliol poliéster y las bandas en el rango de los 1007$675 \mathrm{~cm}^{-1}$ corresponden a los las flexiones de los grupos alquenilos que se encuentran presentes dentro de la estructura del aceite de ricino (Mazo, 2006) y en la región de1700-1530 $\mathrm{cm}^{-1}$, corresponde a la vibración causada por la flexión de las moléculas de agua contenidas en la red o bien un exceso de silanol (Velásquez, 2000) que se generaron en la reacción del PU y a la policondensación del proceso sol-gel.

Las bandas de absorción a 3450, 1731, 1638 y $1020 \mathrm{~cm}^{-1}$ corresponden al enlace $\mathrm{N}-\mathrm{H}$, $\mathrm{O}-\mathrm{H}$, carbonilo de éster $(\mathrm{O}-\mathrm{C}=\mathrm{O})$, carbonilo de amida $(\mathrm{NH}-\mathrm{C}=\mathrm{O})$ y la frecuencia de estiramiento O-C. Estas son las bandas caracteristicas de enlace del grupo uretano, $\mathrm{NH}-(\mathrm{C}=\mathrm{O})-\mathrm{O}-($ Ghosh, 2018).

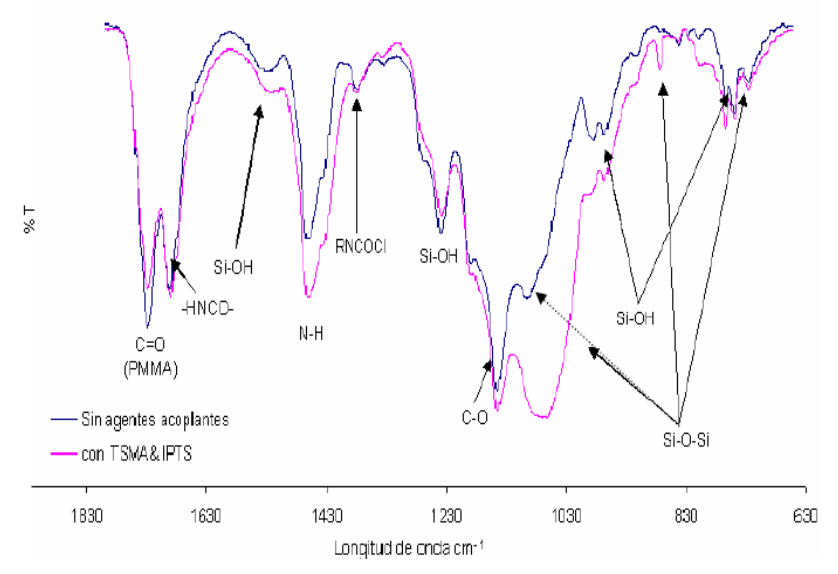

Figura 4 Espectro FTIR para el sistema PU/PMMA/SiO 2 en un intervalo de 1830- $630 \mathrm{~cm}^{-1}$

Fuente: Elaboración Propia

\section{Agradecimiento}

Agradecemos las facilidades otorgadas al Instituto Tecnológico de Ciudad Madero del Tecnológico Nacional de México para el desarrollo de este proyecto.

\section{Conclusiones}

La realización de las redes binarias, determinaron los tiempos y condiciones de reacción para la síntesis del sistema ternario, en donde los factores ambientales afectaron en la obtención de algunas de ellas. En los estudios de FTIR se observaron los grupos representativos de cada sistema, en donde la aparición de diversas bandas atribuidas al silanol ( $\mathrm{Si}-\mathrm{OH})$ indican que no se obtuvo una conversión completa sino parcial de sílica.

Los grupos característicos de los sistemas individuales $\left(\mathrm{SiO}_{2}\right)$, binarios y ternarios de cada red fueron determinados mediante esta técnica. El uso de IPTS y MSMA funcionalizan la IPN ternaria, lo cual indica que se tiene mayor compatibilidad en la red híbrida; aunque en el espectro infrarrojo de la muestra se observa un pico atribuido a la poliurea, la IPN es muy transparente. Sin mebrago, existe la posibilidad de que las IPNS`s puedan ser trabajadas, con otros agentes acoplantes, que permitan obtener un material mas resisten al impacto.

\section{Referencias}

Babonneau, F., Sanchez C., (1996) "Stratégies de Synthèse des Matériaux Hybrides“, ed. Masson, Paris, 33-55.

Bonilla, M., G. A., (2005) Tesis de doctorado: "Réseaux Hybrides Interpénétrés", Instituto Charles Sadron, Estrasburgo, Francia, 5-15, 7789, 115-150.

Chenal, J.M., (2002) Tesis de doctorado: "De l'importance des conditions de synthèse sur la modification de l'interphase d'un système interpénétré de deux polymères réticulés", Estrasburgo, Francia.

Dave, V. J., Patel, H. S., (2013), "Synthesis and characterization of interpenetrating polymer networks from transesterified castor oil based polyurethane and polystyrene", 1-7.

Djomo, H., (1982) Tesis de doctorado: "Réseaux polymers interpénétrés à base de polyuréthane et de polyméthacrylate de méthyle: Synthèse, caractérisation, propriétés", Instituto Charles Sadron, Estrasburgo, Francia. 
Ghosh, T., Karak, N., (2018) “Tough interpenetrating polymer network of silicone containing polyurethane and polystyrene with self-healing, shape memory and self-cleaning attributes" Royal Society of Chemistry.

Hahn, W., (1985) "Polyurethane Handbook", 2ed., Hanser, Munich,11-16, 33-37, 55-56, 6569.

Kausar, A., 2019 "Interpenetrating polymer network and nanocomposite IPN of polyurethane/epoxy: a review on fundamentals and advancements" Polymer-Plastics Technology and Materials.

He, X., Widmaier, J. M., Meyer, G. C., (1993) Artículo: "Kinetics Of Phase Separation in Polyurethane /Polystyrene Semi-1 Interpenetrating Polymer Networks. 1.Light Transmission Studies", Polymer International, Vol. 32, 289-293.

Liang, J., Deng J., (2017), "Chiral Interpenetrating Polymer Network Constructed by Helical Substituted Polyacetylenes and Used for Glucose Adsorption", 1-7.

Mazo, P. C., Restrepo, G., Estenoz, D., (2006 ) "Síntesis y caracterización de espumas de Poliuretano utilizando aceites vegetales: XIV Congreso colombiano de química".

Olvera, I.V., Armenta, R.J.L., Antonio, C.R.C., Mendoza, M.A.M, (2007) Artículo: "Comportamiento térmico de IPN's de PU / PMMA modificados con cargas inorgánicas", Revista Iberoamericana de Polímeros, vol. 8(4), p. 313-322

Pretsch, E., Cler, T., Seibl, J., Simon, (1996,) "Tablas para la elucidación estructural de compuestos orgánicos por métodos espectroscópicos", Alambra Longman, España, I.5 - I.250.

Sanchez C., (1996) "Les Matériaux Hybrides: Définition et Classification“. Ed. Masson, Paris, 27-31.

Sanchez C., (1996) "Matériaux Hybrides: Introduction in Matériaux Hybrides", Ed. Masson, Paris, 17-19.
Velázquez, G., Martín, M.O, (2000) "Cuantificación por IR de agua directamente unida a una matriz polimérica", Sociedad Mexicana de Ciencia de Superficies y de Vacío, 11, 24-31.

Wingrove, A. S., Caret, R. L., (1981) "Química orgánica: espectroscopía infrarroja”, 559-571. 